\title{
MHD COMPRESSIVE TURBULENCE IN THE SOLAR WIND AND THE NEARLY INCOMPRESSIBLE APPROACH
}

\author{
B. BAVASSANO and R. BRUNO \\ Istituto di Fisica dello Spazio Interplanetario, CNR \\ via G. Galilei, CP 27, 00044 Frascati, Italy \\ and \\ H. ROSENBAUER \\ Max-Planck-Institut für Aeronomie \\ Postfach 20, D-37189, Katlenburg-Lindau, Germany
}

\begin{abstract}
At MHD scales density fluctuations in the solar wind generally have a relative amplitude less than 0.1 . The nearly incompressible MHD theory would seem then appropriate to describe a major part of the compressive turbulence at these scales. As a test of the theory, we focus on the scaling properties of density fluctuations with turbulent Mach numbers and on the level of correlation between density and temperature fluctuations. Our findings do not appear in favour of an extended applicability of the nearly incompressible theory to MHD compressive turbulence in the solar wind.
\end{abstract}

Key words: Solar Wind - MHD Turbulence - Density Fluctuations

\section{Introduction}

Compressive fluctuations at magnetohydrodynamic (MHD) scales generally exhibit a small amplitude and carry noticeably less energy than other fluctuations. It has been suggested that they might well be described by the nearly incompressible (NI) MHD theory, developed in recent years (see Zank and Matthaeus, 1992 and references therein). Two different approaches are possible. One is that of the heat-fluctuation-modified (HFM) fluids, where relative pressure, density, and temperature fluctuations are all on an equal footing and scale with the turbulent Mach number (M) as $\mathrm{M}^{2}$. For such fluids the correlation between density $(\mathrm{N})$ and temperature $(\mathrm{T})$ fluctuations is positive, provided that acoustic and incompressible pressure fluctuations are correlated with temperature fluctuations. The second case is that of the heat-fluctuation-dominated (HFD) fluids, characterized by relatively stronger fluctuations in density and temperature (both scaling as $\mathrm{M}$ ) compared with those in thermal pressure (scaling as $\mathrm{M}^{2}$ ). For HFD fluids the $\mathrm{N}$ - $\mathrm{T}$ correlation is negative, in sharp contrast with the HFM case.

Experimental tests of the NI theory recently performed by Bavassano, Bruno and Klein (1995) and by Bavassano and Bruno (1995) are briefly described here. The dependence of the relative density fluctuations $(\delta \mathrm{N} / \mathrm{N})$ upon $\mathrm{M}$ and the level of correlation between $\mathrm{N}$ and $\mathrm{T}$ fluctuations have been investigated. Helios 2 plasma data in the inner heliosphere (0.3 to $1 \mathrm{AU})$ 


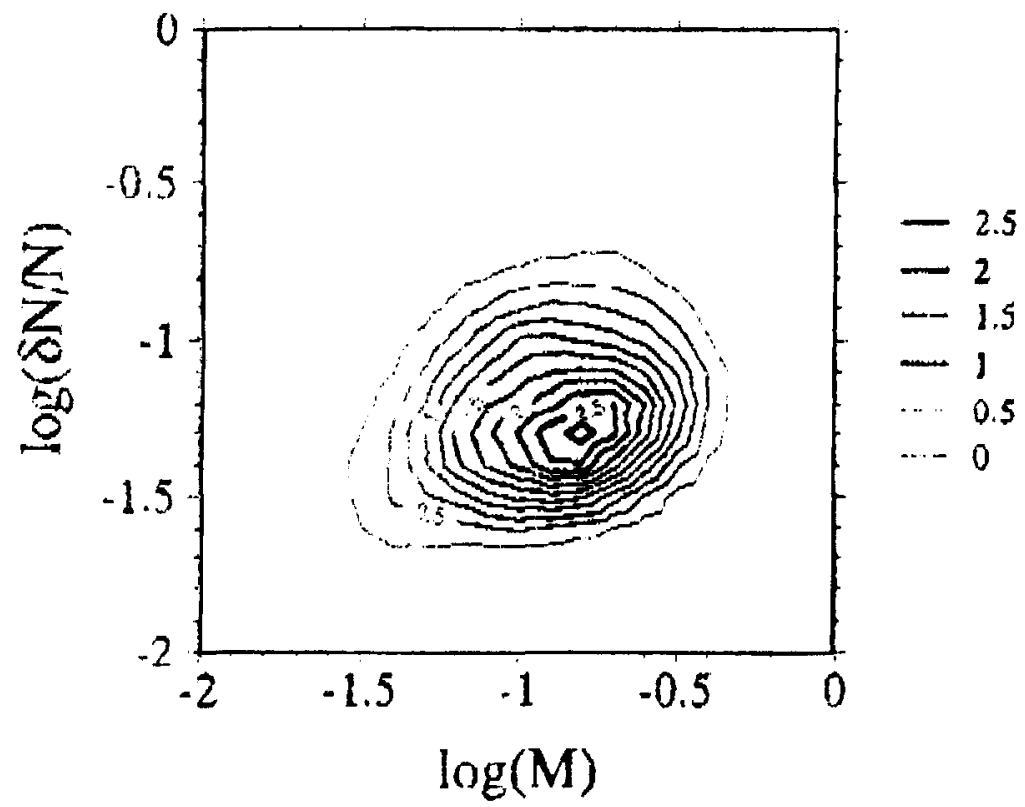

Fig. 1. Number-of-cases (in percent) distribution on the $\log (M)-\log (\delta N / N)$ plane.

for a period of about three months at low solar activity have been used, $\mathrm{N}$ and $\mathrm{T}$ fluctuations and $\mathrm{M}$ values have been determined on 45 -min intervals, with $\mathrm{M}$ given by the velocity fluctuations to sound speed ratio.

\section{Observations}

Frequency distributions of both $\delta \mathrm{N} / \mathrm{N}$ and $\mathrm{M}$ have been determined. In about $86 \%$ of cases $\delta \mathrm{N} / \mathrm{N}$ has values below 0.1 , confirming that compressive phenomena are not strong at the investigated scales. Turbulent Mach numbers are distributed around a most probable value slightly greater than 0.1 , with an extended tail to lower values. Thus, for a major fraction of the examined sample, both $\delta \mathrm{N} / \mathrm{N}$ and $\mathrm{M}$ appear small enough to make applicable, in principle, the NI approach. Figure 1 shows how $\delta \mathrm{N} / \mathrm{N}$ varies with $M$. It is clearly apparent that neither a scaling as M (HFD case) nor as $M^{2}$ (HFM case) emerges from the observed distribution.

Linear correlation coefficients and best fit straight lines have been determined for the entire sample and for various subsets of it at given ranges of solar wind speed (V) and heliocentric distance (R). In the light of the NI theory, a lower (in absolute value) threshold of 0.6 for the correlation coefficient $\rho_{\mathrm{NT}}$ between $\mathrm{N}$ and $\mathrm{T}$ fluctuations has also been introduced. Results 
TABLE I

$\log (\delta \mathrm{N} / \mathrm{N})$ Versus $\log (\mathrm{M})$ : Linear Fit and Correlation

\begin{tabular}{rrrrrr}
\hline $\mathrm{V}(\mathrm{km} / \mathrm{s})$ & $\mathrm{R}(\mathrm{AU})$ & $\rho_{\mathrm{NT}}$ & \multicolumn{1}{c}{ Slope } & $\rho$ & Data \\
\hline all & all & all & $0.21 \pm 0.01$ & 0.25 & 4693 \\
all & all & all & $0.20 \pm 0.01$ & 0.23 & 4459 \\
$<500$ & all & all & $0.40 \pm 0.02$ & 0.42 & 1929 \\
$>550$ & all & all & $0.21 \pm 0.02$ & 0.25 & 2219 \\
$<500$ & $<0.5$ & all & $0.26 \pm 0.04$ & 0.32 & 384 \\
$<500$ & $>0.8$ & all & $0.41 \pm 0.03$ & 0.42 & 1022 \\
$>550$ & $<0.5$ & all & $0.15 \pm 0.04$ & 0.15 & 608 \\
$>550$ & $>0.8$ & all & $0.16 \pm 0.03$ & 0.18 & 979 \\
all & all & $<-0.6$ & $0.20 \pm 0.07$ & 0.23 & 171 \\
$<500$ & all & $<-0.6$ & $0.21 \pm 0.07$ & 0.24 & 160 \\
$>550$ & all & $<-0.6$ & $0.10 \pm 0.33$ & 0.14 & 7 \\
$<500$ & $<0.5$ & $<-0.6$ & $-0.01 \pm 0.10$ & -0.02 & 51 \\
$<500$ & $>0.8$ & $<-0.6$ & $0.31 \pm 0.10$ & 0.34 & 69 \\
$>550$ & $<0.5$ & $<-0.6$ & & & 0 \\
$>550$ & $>0.8$ & $<-0.6$ & $-0.44 \pm 0.45$ & -0.57 & 4 \\
all & all & $>0.6$ & $0.15 \pm 0.04$ & 0.18 & 507 \\
$<500$ & all & $>0.6$ & $0.35 \pm 0.08$ & 0.33 & 159 \\
$>550$ & all & $>0.6$ & $0.18 \pm 0.05$ & 0.22 & 305 \\
$<500$ & $<0.5$ & $>0.6$ & $0.94 \pm 0.20$ & 0.74 & 20 \\
$<500$ & $>0.8$ & $>0.6$ & $0.39 \pm 0.12$ & 0.32 & 100 \\
$>550$ & $<0.5$ & $>0.6$ & $0.02 \pm 0.09$ & 0.02 & 129 \\
$>550$ & $>0.8$ & $>0.6$ & $0.27 \pm 0.09$ & 0.29 & 106 \\
\hline
\end{tabular}

are summarized in Table I. The first three columns indicate the allowed ranges for $\mathrm{V}, \mathrm{R}$, and $\rho_{\mathrm{NT}}$, respectively. In the fourth and fifth columns the slope of the best fit line and the correlation coefficient $\rho$ for the examined $[\log (\mathrm{M}), \log (\delta \mathrm{N} / \mathrm{N})]$ sample are reported, respectively. Last column gives the number of the examined 45-min intervals. Results in the first row refer to the complete data sample, while those given in the other rows have been derived with an upper limit of -0.5 for both $\log (\delta N / N)$ and $\log (M)$, to reject unusually large variations in density and velocity.

Table I shows that in the analysed samples a significant linear correlation between $\log (\delta \mathrm{N} / \mathrm{N})$ and $\log (\mathrm{M})$ is not found. For cases without any constraint on $\rho_{\mathrm{NT}}$ the highest value of $\rho$ is around 0.4. Results are even worse for cases with $\rho_{\mathrm{NT}}<-0.6$. For $\rho_{\mathrm{NT}}>0.6$ the only significant value of $\rho(0.74)$ comes from a very small sample and the corresponding slope (0.94) does not match the $\mathrm{M}^{2}$ scaling expected for positive $\rho_{\mathrm{NT}}$ (HFM fluid). 
The poor correlation may derive from the small dynamic range of the investigated parameters. A range extension can only be obtained by decreasing the lower limit of the variability interval, if the NI theory test has to remain meaningful. This is not easily feasible in the near-ecliptic solar wind, that does not appear homogeneous enough. More favourable conditions should be offered by high-latitude observations. On the other hand, a narrow range for the parameters involved in NI theory might be an intrinsic feature of the interplanetary plasma. Thus, instead of looking for M scalings, a better way of testing the theory should be one based on an analysis of the correlation between $\mathrm{N}$ and $\mathrm{T}$ fluctuations.

We determined the distribution of $\rho_{\mathrm{NT}}$ versus the solar wind velocity. Cases with $\rho_{\mathrm{NT}}<-0.8$ are $\sim 1.0 \%$ of the investigated samples. They are mainly observed in slow wind. Conversely, cases with $\rho_{\mathrm{NT}}>0.8$ occur almost independently on the flow speed. They amount to $\sim 1.5 \%$ of all analysed cases. This very low occurrence of strong $\mathrm{N}-\mathrm{T}$ correlations (positive or negative) does not fit well in the view proposed by the NI theory.

\section{Conclusion}

Observations are not in favour of an extended applicability of the NI theory to MHD compressive fluctuations in the inner solar wind. A likely explanation is that generally the solar wind is not homogeneous enough to make applicable the NI theory. In an alternative view, a relevant role in generating compressive turbulence may be played by pressure imbalances between contiguous flow-tubes, with cascade processes to smaller scales (Marsch and $\mathrm{Tu}, 1994)$. This last issue, however, needs further investigations.

\section{Acknowledgements}

We gratefully acknowledge fruitful discussions with L. W. Klein. This work has been partially supported by the Italian Space Agency (ASI).

\section{References}

Bavassano, B. and Bruno, R.: 1995, 'Density fluctuations and turbulent Mach numbers in the inner solar wind', J. Geophys. Res., in press

Bavassano, B., Bruno, R. and Klein, L. W.: 1995, 'Density-temperature correlation in solar wind MHD fluctuations: A test for nearly incompressible models', J. Geophys. Res. , in press

Tu, C.-Y. and Marsch, E.: 1994, 'On the nature of compressive fluctuations in the solar wind', J. Geophys. Res. 99, 21,481-21,509

Zank, G. P. and Matthaeus, W. H.: 1992, 'Waves and turbulence in the solar wind', J. Geophys. Res. 97, 17,189-17,194 